\title{
Effect of ACE2 Expression Inhibiting Drugs on COVID-19 Disease Severity, Outcome and Length of Admission in Ethiopian Patients: A Causal Inference Using Marginal Structural Model with Inverse Probability Weight
}

Tigist W. Leulseged ( $\nabla$ tigdolly@gmail.com )

St. Paul's Hospital Millennium Medical College Ishmael S. Hassen

St. Paul's Hospital Millennium Medical College Wuletaw C. Zewde

St. Paul's Hospital Millennium Medical College Endalkachew H. Maru

St. Paul's Hospital Millennium Medical College Lydia K. Naylor

St. Paul's Hospital Millennium Medical College Yakob G. Tsegay

St. Paul's Hospital Millennium Medical College Mesay G. Edo

St. Paul's Hospital Millennium Medical College Naol A. Baruda

St. Paul's Hospital Millennium Medical College

Fiseha E. Mihretu

St. Paul's Hospital Millennium Medical College

Zerihun A. Terefe

St. Paul's Hospital Millennium Medical College

Nardos T. Kidane

St. Paul's Hospital Millennium Medical College Henok N. Desalegn

St. Paul's Hospital Millennium Medical College Dawit A. Abebe

St. Paul's Hospital Millennium Medical College 
Keywords: COVID-19, ACEls, ARBs and/or NSAIDs, retrospective cohort, causal inference, Ethiopia

Posted Date: July 29th, 2021

DOI: https://doi.org/10.21203/rs.3.rs-724357/v1

License: (c) (1) This work is licensed under a Creative Commons Attribution 4.0 International License. Read Full License 


\section{Abstract \\ Background}

The role of drugs that inhibit ACE2 expression on COVID-19 disease severity, progression and outcome has been debatable with studies reporting contradictory findings. So far, there is no such study conducted in Africa. Having clarity on this issue is relevant as these drugs are the commonly prescribed medications for patients with co-morbid illnesses who are reported to be vulnerable to COVID-19 poor outcome. Therefore, the aim of this study was to assess the effect of acute or chronic ACEls, ARBs and/or NSAIDs use on COVID-19 disease severity, outcome and length of admission among patients with COVID-19 admitted to the Millennium COVID-19 Care Center in Ethiopia.

\section{Methods}

A retrospective cohort study was conducted among 945 patients with COVID-19 who were on follow up from July 2nd to December 25th, 2020. Data was described using frequency tables and cross tabulations. To identify the effect of ACEIs, ARBs and/or NSAIDs use on COVID-19 disease severity, disease outcome and length of admission, Marginal Structural Model (MSM) with inverse probability weighting (IPW) approach was used.

\section{Results}

Among the 945 patients studied, 115 (12.2\%) had a history of ACEls, ARBs and/or NSAIDs use. At admission, the majority (39.6\%) had mild disease and 272 (28.8\%) had severe disease. Among the study participants, 900 (95.2\%) were discharged improved and the rest 45 (4.8\%) died. The median length of admission was 14.0 days (IQR, 13-16). Multinomial Logistic Regression, Log Binomial Regression and Negative Binomial Regression models were fitted to assess the effect of ACEls, ARBs and/or NSAIDs use on disease severity, outcome and length of admission respectively. In all the three outcome models, ACEIs, ARBs and/or NSAIDs use didn't show a statistically significant association with the outcomes.

\section{Conclusions}

Acute or chronic use of ACEls, ARBs and/or NSAIDs showed no effect on COVID-19 disease severity, outcome and length of admission and therefore should not be withdrawn from patients who need these therapies.

\section{Background}

The Coronavirus pandemic has affected the entire world causing a tremendous loss to human life and also caused a burden to the existing health care system making it difficult to provide the best care 
possible for a better outcome especially in the developing countries. Because of this, the patient admission, treatment and discharge criteria has been continually improved by the World Health Organization (WHO) to accommodate the most in need cases to the health care provision system. To that end, risk stratification based on disease presentation, severity, patient characteristics, existing medical conditions and drug intake history has been given great importance. Therefore, providing preventive services and strict observation for high risk groups should be strictly applied to prevent deterioration and complication at which point the care provided might not bring favorable results. With this aim, different researches were conducted with results showing geographical disparity and also inconsistency even in similar setups calling for the need for more research to be conducted especially in African setup with limited research reports on COVID-19 so far.

Among the proposed important predictors of COVID-19 disease progression and outcome is a history of taking drugs that inhibit the expression of Angiotensin-converting enzyme 2 (ACE2). This is proposed because of the pathological process of the SARS-CoV-2 virus entry into the body using ACE2. Therefore, taking these type of drugs (ACE inhibitors (ACEls), angiotensin receptor blockers (ARBs) and nonsteroidal anti-inflammatory drugs (NSAIDs)) increase the level of ACE2 and in turn increasing the possibility of the virus to enter the body. Therefore, to better understand the effect of these drugs on disease progression and outcome (hospital/ ICU admission, length of hospital stay, complications, need for mechanical ventilation and mortality), different studies in different countries were conducted reporting contradicting results.

Systematic review and meta-analysis reports demonstrated that there is no increased risk of any of the disease related complication or outcome in those with ACEls and ARBs intake history [1-3]. In addition, NSAIDs were reported to have no effect on disease progression and outcome in another systematic review [4]. Similarly, studies conducted in Italy, China, Korea, Spain and the United States show that the use of ACEIs, ARBs and NSAIDs did not affect disease progression, complication and outcome [5-10].

On the contrary, a study conducted in Turkey and Saudi Arabia reported that ACEls and ARBs therapy were associated with higher risk of severe or critical COVID-19 disease, need of ICU care and higher incidence of in-hospital death [11, 12]. As opposed to this, a study conducted in Kuwait showed that use of ACEls and ARBs is inversely associated with ICU admission and mortality implying that these drugs have a protective effect from adverse disease outcome [13].

ACEIs, ARB s and NSAIDs are widely used drugs for the treatment of chronic conditions which are a problem of the developing countries, as much as it is a developed countries problem, showing an increasing trend in recent years with a larger proportion (77\%) of deaths reported from low- and middleincome countries [14]. With such varying and contradicting reports and in the face of lack of evidence generated on the effect of these drugs on the disease in the African population, clinical judgement to continue or discontinue these life-saving medications should solely rely on evidence generated from the local population. 
Therefore, the aim of this study was to assess the effect of acute or chronic ACEls, ARBs and/or NSAIDs use on COVID-19 disease severity, outcome and length of admission among patients with COVID-19 admitted to the Millennium COVID-19 Care Center in Ethiopia from July 2nd to December 25th, 2020.

\section{Methods}

\section{Study setting, design and population}

An institution-based retrospective cohort study was conducted at Millennium COVID-19 Care Center (MCCC), a makeshift hospital in Addis Ababa, Ethiopia dedicated for isolating and treating COVID-19 cases only. It is the largest Center in the country with a large flow of patients. The admission pattern initially included all types of patients who tested positive for SARS-Cov-2 with the aim of controlling the pandemic.

The follow up was made from July 2nd to December 25th, 2020. The source population was all cases of COVID-19 admitted at MCCC with a confirmed diagnosis of COVID-19 using RT-PCR, as reported by a laboratory given mandate to test such patients by the Ethiopian Federal Ministry of Health and who were on follow up from July 2nd to December 25th, 2020 [15].

All consecutively admitted patients with COVID-19 during the follow up period and who consent to participate were included in the study. With these criteria, a total of 945 patients with COVID-19 were included in the final analysis.

\section{Eligibility criteria}

All patients with COVID-19 who were on treatment and follow up at the center from July 2nd to December 25th, 2020 and who consent to participate were included.

\section{Data Collection Procedures and Quality Assurance}

Data was extracted from patients' admission, follow up and discharge medical records using a pretested electronic data abstraction tool that is adopted from the WHO CRF form by trained data collectors. Appropriate infection prevention and control measures were followed during the data collection process. Data quality was further assured through double data entry, and data cleaning through checking for inconsistencies, numerical errors and missing parameters. Where discrepancies are observed, data entered was verified with the primary data source. Once data cleaning was complete, data was exported to STATA software version 14 (College Station, TX) for analysis.

\section{Statistical Analysis}

Data was summarized using frequency tables and percentages. To compare the socio-demographic and clinical characteristics between the two groups (ACEls, ARBs and/or NSAIDs users Vs Non users), Chisquare test, Fischer's exact test and independent t-test were used. 
To identify the effect of ACEls, ARBs and/or NSAIDs use on COVID-19 disease severity (mild vs moderate vs severe), disease outcome (alive vs dead) and length of admission (in days), Marginal Structural Model (MSM) with inverse probability weighting (IPW) approach was used.

\section{Treatment model}

The treatment model that uses binary logistic regression was fitted to estimate the probability of exposure given the covariates (propensity score). The estimated probability of exposure was used to compute the inverse probability weights for each individual. The inverse of the probability of exposure was then used to weight each individual in the estimation of the marginal odds ratio. Variables to be included in the final treatment model were selected by univariate analysis at $25 \%$ level of significance and also based on the existing literature reviewed.

\section{Outcome models}

There are three outcome variables in this study; disease severity (mild vs moderate vs severe), disease outcome (alive vs dead) and length of admission (in days). All the three outcomes were predicted by including the treatment variable alone in the respective models after adjusting for inverse probability weights.

To identify the effect of treatment on COVID-19 disease severity, Multinomial Logistic Regression model was used where adjusted relative risk (ARR), P-value and 95\% $\mathrm{Cl}$ for ARR were used to test the presence of statistically significant relationship.

To identify the effect of treatment on COVID-19 disease outcome, Log Binomial Regression model was used where adjusted relative risk (ARR), P-value and 95\% $\mathrm{Cl}$ for ARR were used to test the presence of statistically significant relationship.

To identify the effect of treatment on length of admission, Negative Binomial Regression model was used where adjusted relative risk (ARR), P-value and $95 \% \mathrm{Cl}$ for ARR were used to test the presence of statistically significant relationship. Negative binomial Poisson regression model was used instead of Standard Poisson regression model because the assumption of Standard Poisson regression model (mean equals variance) was checked and there was over dispersion depicted by comparison of mean and variance of the outcome variable and confirmed by the significance of dispersion parameter. And finally Model fitness was checked for the Negative binomial Poisson regression model using Pearson chi square and deviance tests and the model fits the data well.

In all the three models, with a $p$-value of $\leq 0.05$, the treatment was considered as a significant predictor of disease severity.

\section{Results}

\section{Socio-demographic and clinical characteristics}


The median age of the participants was 41 (IQR, 29-58) years. More than half of the participants were males $(60.6 \%)$. Four hundred thirteen $(43.7 \%)$ had a history of one or more preexisting co-morbid illness. The majority had hypertension (26.9\%) followed by Type II diabetes mellitus (TIIDM) (18.5\%) and Asthma (5.5\%). The most common reported symptoms were cough (52.9\%), SOB (27.1\%), fatigue $(24.4 \%)$ and fever (21.8\%).

One hundred fifteen (12.2\%) had a history of ACEls, ARBs and/ or NSAIDs use. At admission, the majority (39.6\%) had mild disease and $272(28.8 \%)$ had severe disease. Among the study participants, 900 (95.2\%) were discharged improved and the rest 45 (4.8\%) died. The median length of admission was 14.0 days (IQR, 13-16). (Table 1)

\section{Comparison of socio-demographic and clinical characteristics based on drug use history}

Based on the chi-square or Fischer's exact test and independent t-test result, a significant difference between those who has a history of ACEls, ARBs and/or NSAIDs use and those who don't showed that the two groups showed a significant difference in age category, the presence of shortness of breath, disease severity, outcome and length of admission.

Accordingly, a significantly higher proportion of patients who has a history of ACEls, ARBs and/or NSAIDs use history are in the age range of $50-59$ years $(27.0 \%$ Vs $11.3 \%$, p-value $<0.0001)$ and 60 years and older $(47.0 \%$ Vs $20.4 \%$, p-value $<0.0001)$ compared to those with no drug use history.

A significantly higher proportion of patients with a history of ACEIs, ARBs and/or NSAIDs use has a shortness of breath at presentation compared to those with no drug use history $(41.7 \% \%$ Vs $25.1 \%$, pvalue<0.0001).

A significantly higher proportion of patients with a history of ACEls, ARBs and/or NSAIDs use had severe COVID-19 disease at presentation compared to those with no drug use history (47.0\% \% Vs $26.3 \%$, pvalue $<0.0001$ ). Similarly, a significantly smaller proportion of patients with a history of ACEIs, ARBs and/or NSAIDs use had mild and moderate COVID-19 disease at presentation compared to those with no drug use history ( $26.1 \% \%$ Vs $41.4 \%$, p-value $<0.0001$ for mild disease and $27.0 \%$ Vs $32.3 \%$, pvalue<0.0001).

A significantly higher proportion of patients with a history of ACEls, ARBs and/or NSAIDs use died of COVID-19 compared to those with no drug use history (9.6\% \% Vs $4.1 \%$, p-value $=0.010)$.

A statistically significant difference was observed in the length of admission where having a history of ACEIs, ARBs and/or NSAIDs use was associated with a delayed recovery compared to those with no drug use history (14.5 days Vs 14.4 days, $p$-value $=0.002$ ). But, this difference might not have a significant clinical implication. (Table 2) 


\section{Treatment model: Logistic regression of factors affecting use of ACEls, ARBs and/or NSAIDs}

The treatment model using a binary logistic regression model was run by including variables that were significant on univariate analysis at $25 \%$ level of significance and also from variables selected to be useful based on literature review. Accordingly, age category, sex, cardiac illness, hypertension, TIIDM, asthma, fever, cough, sore throat, runny nose, chest pain, myalgia, arthralgia, fatigue, shortness of breath and headache were included in the final treatment model. By fitting the final treatment model, propensity score was estimated and it was used to compute the inverse probability weights for each individual. The inverse of the probability of exposure was then used to weight each individual in the estimation of the marginal odds ratio. (Table 3 )

Table 3: Treatment model: Binary Logistic Regression model of factors affecting use of ACEIs, ARBs and/or NSAIDs $(n=945)$

\begin{tabular}{|c|c|c|c|}
\hline Variables & AOR & 95\% CI for AOR & p-value \\
\hline \multicolumn{4}{|l|}{ Age category (in years) } \\
\hline$<30$ & 1 & 1 & \\
\hline $30-39$ & 2.07 & $0.58,7.32$ & 0.261 \\
\hline $40-49$ & 1.69 & $0.49,5.73$ & 0.398 \\
\hline $50-59$ & 3.82 & $1.14,12.79$ & $0.030 *$ \\
\hline$\geq 60$ & 1.89 & $0.57,6.37$ & 0.300 \\
\hline Male sex (Vs Female) & 1.83 & $1.08,3.11$ & $0.025 *$ \\
\hline Cardiac illness & 9.95 & $4.69,21.09$ & $<0.0001 *$ \\
\hline Hypertension & 14.87 & $7.89,28.01$ & $<0.0001 *$ \\
\hline Type II Diabetes Mellitus & 1.52 & $0.88,2.64$ & 0.137 \\
\hline Asthma & 1.69 & $0.66,4.32$ & 0.278 \\
\hline Fever & 0.69 & $0.33,1.44$ & 0.323 \\
\hline Cough & 0.57 & $0.31,1.04$ & 0.065 \\
\hline Sorethroat & 1.06 & $0.46,2.43$ & 0.895 \\
\hline Runny nose & 1.40 & $0.47,4.18$ & 0.543 \\
\hline Chest pain & 1.49 & $0.69,3.25$ & 0.313 \\
\hline Myalgia & 1.31 & $0.53,3.22$ & 0.558 \\
\hline Arthralgia & 0.62 & $0.23,1.72$ & 0.360 \\
\hline Fatigue & 0.53 & $0.25,1.12$ & 0.095 \\
\hline Shortness of breath & 1.67 & $0.87,3.19$ & 0.121 \\
\hline Headache & 1.55 & $0.75,3.24$ & 0.239 \\
\hline
\end{tabular}


Note: AOR, Adjusted Odds ratio; CI, Confidence interval; *statistically significant Table 3: Treatment model: Binary Logistic Regression model of factors affecting use of ACEIs, ARBs and/or NSAIDs ( $\mathrm{n}=945)$

\section{Outcome Model: Effect of ACEls, ARBs and/or NSAIDs use on disease severity, outcome and length of admission}

Three outcome models; Multinomial Logistic Regression, Log Binomial Regression and Negative Binomial Regression models were fitted to assess the effect of ACEls, ARBs and/or NSAIDs use on disease severity, outcome and length of admission respectively. To predict all the three outcomes, the treatment variable alone was fitted as an explanatory variable after adjusting for inverse probability weights.

Accordingly, on the three outcome models, ACEls, ARBs and/or NSAIDs use didn't show a statistically significant association with all the three outcomes at $5 \%$ level of significance. (Table $\mathbf{4}, \mathbf{5}$ and $\mathbf{6}$ )

Table 4: Multinomial logistic regression of Effect of ACEIs, ARBs and/or NSAIDs use on disease severity $(n=945)$

\begin{tabular}{|c|c|c|c|c|}
\hline \multirow{2}{*}{ Variable } & \multicolumn{2}{|c|}{ Moderate (Vs Mild) } & \multicolumn{2}{c|}{ Severe (Vs Mild) } \\
\cline { 2 - 5 } & ARR (95\% CI) & P-value & ARR (95\% CI) & P-value \\
\hline ACEIs, ARBs and/or NSAIDs & & & & \\
\hline No & 1 & & 1 & \\
\hline Yes & $0.76(0.25,2.31)$ & 0.628 & $1,21(0.45,3.27)$ & 0.708 \\
\hline
\end{tabular}

Note: ARR, Adjusted Relative Risk; CI, Confidence interval; *statistically significant Table 4: Multinomial logistic regression of Effect of ACEIs, ARBs and/or NSAIDs use on disease severity $(n=945)$

Table 5: Log binomial regression of Effect of ACEIs, ARBs and/or NSAIDs use on disease outcome $(\mathrm{n}=945)$

\begin{tabular}{|c|c|c|}
\hline Variable & ARR (95\% CI) & P-value \\
\hline ACEIs, ARBs and/or NSAIDs & & \\
\hline No & 1 & \\
\hline Yes & $1.14(0.27,4.82)$ & 0.861 \\
\hline
\end{tabular}

Note: ARR, Adjusted Relative Risk; CI, Confidence interval; *statistically significant 
Table 5: Log binomial regression of Effect of ACEIs, ARBs and/or NSAIDs use on disease outcome $(n=945)$

Table 6: Negative binomial regression of Effect of ACEIs, ARBs and/or NSAIDs use on length of admission $(n=945)$

\begin{tabular}{|c|c|c|}
\hline Variable & ARR (95\% CI) & P-value \\
\hline ACEIs, ARBs and/or NSAIDs & & \\
\hline No & 1 & \\
\hline Yes & $0.99(0.88,1.11)$ & 0.842 \\
\hline
\end{tabular}

Note: ARR, Adjusted Relative Risk; CI, Confidence interval; *statistically significant Table 6: Negative binomial regression of Effect of ACEIs, ARBs and/or NSAIDs use on length of admission $(n=945)$

\section{Discussion}

In this study, we assessed the effect of acute or chronic ACEls, ARBs and/or NSAIDs use on COVID-19 disease severity, outcome and length of admission among patients with COVID-19 admitted to the Millennium COVID-19 Care Center in Ethiopia from July 2nd to December 25th, 2020. To our knowledge, this is the first study conducted in the African set up. Understanding this helps (provides an input) in modifying the risk stratification, prevention and admission practices so that better patient outcome can be achieved.

One hundred fifteen (12.2\%) had a history of ACEls, ARBs and/or NSAIDs use. At admission, the majority (39.6\%) had mild disease and $272(28.8 \%)$ had severe disease. Among the study participants, 900 $(95.2 \%)$ were discharged improved and the rest $45(4.8 \%)$ died. The median length of admission was 14.0 days (IQR, 13-16).

Based on the chi-square/ Fischer's exact test and independent t-test result, a significant difference between those who has a history of ACEls, ARBs and/or NSAIDs use and those who don't showed that the two groups showed a significant difference in age category, the presence of shortness of breath, disease severity, length of admission and outcome. Accordingly, a significantly higher proportion of patients with a history of ACEls, ARBs and/or NSAIDs were 50 years and older, had shortness of breath at admission, severe disease at presentation, had delayed recovery and died. But on further regression analysis using MSM model with IPW approach, use of ACEIs, ARBs and/or NSAIDs did not show a significant effect on disease severity, outcome and length of admission. Although there are few contradictory reports showing that these drugs have a significant effect on both directions affecting the disease outcome both negatively and positively [11-13], this finding is supported by a number of other institution and community based studies including systematic reviews conducted in non-African setup [1-10]. In addition, a WHO review based on studies conducted outside Africa also showed that there is no wellestablished evidence that patients on these drugs are at higher risk of poor outcome [16]. 
ACEls, ARBs and/or NSAIDs are drugs which are widely used for the treatment of chronic medical conditions. In the current study, chronic medical conditions were reported in 413 (43.7\%) of the participants among which hypertension and cardiac disease, which rely mainly on these drugs for treatment and control, constitutes 313 (75.8\%) of the co-morbid illnesses. In addition, at the global and national level, these conditions are found in a considerable proportion of the general population, implying that the issue of continuing or discontinuing these drugs in patients with COVID-19 will continue to be raised. These medical conditions are also found to be significant determinants of disease severity and outcome among patients with COVID-19 [17-21]. Part of COVID-19 management is stabilizing existing co-morbid conditions so that the body can be at its best immunity for fighting the virus. Therefore, taking these medications is crucial to control the co-morbid conditions which otherwise could exacerbate and lead to progression of the disease ending up in complication and death.

Therefore, the use of these drugs is crucial as part of the management of patients with COVID-19 with comorbid conditions without affecting the COVID-19 disease severity, outcome and length of admission.

\section{Conclusion}

Based on the finding of this study, acute or chronic use of ACEIs, ARBs and/or NSAIDs showed no effect on COVID-19 disease severity, outcome and length of admission. Therefore, we recommend continuation of these drugs for the greater benefit of controlling the co-morbid conditions of patients of any COVID-19 severity.

\section{List Of Abbreviations}

ACE2.........Angiotensin-converting enzyme 2

ACEls.........Angiotensin-converting enzyme inhibitors

ARBs.......... Angiotensin receptor blockers

ARR............. Adjusted relative risk

Cl............... Confidence Interval

COVID-19......... Coronavirus Disease 2019

IPW................ Inverse probability weighting

IQR ................ Interquartile range

NSAIDs.............Nonsteroidal anti-inflammatory drugs

MSM................Marginal Structural Model 
OR Odds Ratio

RT-PCR Real Time Polymerase Chain Reaction

SARS-COV-2......Severe Acute Respiratory Syndrome Coronavirus 2

TIIDM Type II Diabetes Mellitus

WHO World Health Organization

\section{Declarations}

Ethics approval and consent to participate

The study was conducted after obtaining ethical clearance from St. Paul's Hospital Millennium Medical College Institutional Review Board. Written informed consent was obtained from the participants. The study had no risk/negative consequence on those who participated in the study. Medical record numbers were used for data collection and personal identifiers were not used in the research report. Access to the collected information was limited to the principal investigator and confidentiality was maintained throughout the project.

Consent for publication

Not applicable

Availability of data and materials: All relevant data are available upon reasonable request.

Competing interests

The authors declare that they have no known competing interests

Funding: This research did not receive any specific grant from funding agencies in the public, commercial, or not-for-profit sectors.

Authors' Contribution: TWL conceived and designed the study, revised data extraction sheet, performed statistical analysis, and drafted the initial manuscript. ISH contributed in the conception and design of the study, undertook review and interpretation of the data, revised the manuscript and approved the final version. BTA assisted in the statistical analysis, undertook review and interpretation of the data, revised the manuscript and approved the final version. YGT, DSA, MGD, EHM, WCZ, LKN, DFS, MTD and BBT contributed to the conception, obtained patient data, undertook review and interpretation of the data, revised the manuscript and approved the final version.

Acknowledgment 
The authors would like to thank St. Paul's Hospital Millennium Medical College for facilitating the research work.

\section{References}

1. Zhang X, Yu J, Pan L-y, Jiang H-y. ACEI/ARB use and risk of infection or severity or mortality of COVID-19: A systematic review and meta-analysis. Pharmacological Research. 2020;158:104927.

2. van Kimmenade RRJ, Belfroid E, Hoogervorst-Schilp J, Siebelink HJ, Janssen CW, Pinto Y. The effects of ACE2 expression mediating pharmacotherapy in COVID-19 patients. Netherlands Heart Journal. 2021;29(1):20-34.

3. Mackey K, King VJ, Gurley S, Kiefer M, Liederbauer E, Vela K, et al. Risks and Impact of AngiotensinConverting Enzyme Inhibitors or Angiotensin-Receptor Blockers on SARS-CoV-2 Infection in Adults: A Living Systematic Review. Annals of internal medicine. 2020;173(3):195-203.

4. Zhao H, Huang S, Mei K, Shao W, Zhang J, Ma J, et al. Prevalence use of nonsteroidal antiinflammatory drugs in the general population with COVID-19 and associated COVID-19 risk, hospitalization, severity, death, and safety outcomes: A systematic review and meta-analysis. medRxiv. 2021:2021.05.01.21256428.

5. Felice C, Nardin C, Di Tanna GL, Grossi U, Bernardi E, Scaldaferri L, et al. Use of RAAS Inhibitors and Risk of Clinical Deterioration in COVID-19: Results From an Italian Cohort of 133 Hypertensives. American journal of hypertension. 2020;33(10):944-8.

6. Gao C, Cai Y, Zhang K, Zhou L, Zhang Y, Zhang X, et al. Association of hypertension and antihypertensive treatment with COVID-19 mortality: a retrospective observational study. Eur Heart J. 2020;41(22):2058-66.

7. Jung SY, Choi JC, You SH, Kim WY. Association of Renin-angiotensin-aldosterone System Inhibitors With Coronavirus Disease 2019 (COVID-19)- Related Outcomes in Korea: A Nationwide Populationbased Cohort Study. Clinical infectious diseases: an official publication of the Infectious Diseases Society of America. 2020;71(16):2121-8.

8. López-Otero D, López-Pais J, Cacho-Antonio CE, Antúnez-Muiños PJ, González-Ferrero T, Pérez-Poza $M$, et al. Impact of angiotensin-converting enzyme inhibitors and angiotensin receptor blockers on COVID-19 in a western population. CARDIOVID registry. Rev Esp Cardiol (Engl Ed). 2021;74(2):17582.

9. Imam Z, Odish F, Gill I, O'Connor D, Armstrong J, Vanood A, et al. Older age and comorbidity are independent mortality predictors in a large cohort of 1305 COVID-19 patients in Michigan, United States. Journal of internal medicine. 2020;288(4):469-76.

10. Zhou X, Zhu J, Xu T. Clinical characteristics of coronavirus disease 2019 (COVID-19) patients with hypertension on renin-angiotensin system inhibitors. Clinical and experimental hypertension (New York, NY: 1993). 2020;42(7):656 - 60. 
11. Selçuk M, Çınar T, Keskin M, Çiçek V, Kılıç Ş, Kenan B, et al. Is the use of ACE inb/ARBs associated with higher in-hospital mortality in Covid-19 pneumonia patients? Clinical and experimental hypertension (New York, NY: 1993). 2020;42(8):738 - 42.

12. Alrashed AA, Khan TM, Alhusseini NK, Asdaq SMB, Enani M, Alosaimi B, et al. Severity of COVID-19 Infection in ACEl/ARB Users in Specialty Hospitals: A Retrospective Cohort Study. Journal of Infection and Public Health. 2021.

13. ElAbd R, AlTarrah D, AlYouha S, Bastaki H, Almazeedi S, Al-Haddad M, et al. Angiotensin-Converting Enzyme (ACE) Inhibitors and Angiotensin Receptor Blockers (ARB) Are Protective Against ICU Admission and Mortality for Patients With COVID-19 Disease. Frontiers in medicine. 2021;8:600385.

14. World Health Organization. Non-communicable disease report. 13 April 2021.

15. Ethiopian Federal Ministry of Health. Covid19 Management Handbook. 2020.

16. World Health Organization. COVID-19 and the use of angiotensin-converting enzyme inhibitors and receptor blockers. May 7, 2021,.

17. Du R-H, Liang L-R, Yang C-Q, et al. Predictors of mortality for patients with COVID-19 pneumonia caused by SARSCoV-2: a prospective cohort study. The European respiratory journal. 2020;2020(55: 2000524).

18. Gupta S, Hayek SS, Wang W, et al. Factors Associated With Death in Critically III Patients With Coronavirus Disease 2019 in the US. JAMA Intern Med.2020;e203596.

19. Williamson E.J., Walker A.J., Bhaskaran K, et al. Factors associated with COVID-19-related death using OpenSAFELY. Nature.584:430-6 (2020).

20. Xiaochen Li, Shuyun Xu, Muqing Yu, et al. Risk factors for severity and mortality in dult COVID-19 inpatients in Wuhan. The Journal of allergy and clinical immunology. 2020;146:110-8.

21. Leulseged TW, KG A, Hassen IS, et al. COVID-19 Disease Severity and Determinants among Ethiopian Patients: A study of the Millennium COVID-19 Care Center. medRxiv 2020100920209999. 2020.

\section{Tables}

Table 1: Socio-demographic and clinical characteristics $(n=945)$ 


\begin{tabular}{|c|c|c|c|}
\hline Variable & $\begin{array}{l}\text { Frequency } \\
\text { (\%) }\end{array}$ & Variable & $\begin{array}{l}\text { Frequency } \\
\text { (\%) }\end{array}$ \\
\hline Age category (in years) & & Runny nose & \\
\hline$<30$ & $250(26.5)$ & No & $875(92.6)$ \\
\hline $30-39$ & $177(18.7)$ & Yes & $70(7.4)$ \\
\hline $40-49$ & $170(18.0)$ & Chest pain & \\
\hline $50-59$ & $125(13.2)$ & No & $802(84.9)$ \\
\hline$\geq 60$ & $223(23.6)$ & Yes & $143(15.1)$ \\
\hline Sex & & Myalgia & \\
\hline Female & $372(39.4)$ & No & $812(85.9)$ \\
\hline Male & $573(60.6)$ & Yes & $133(14.1)$ \\
\hline $\begin{array}{l}\text { Preexisting Co-morbid } \\
\text { illness }\end{array}$ & & Arthralgia & \\
\hline No & $532(56.3)$ & No & $812(85.9)$ \\
\hline Yes & $413(43.7)$ & Yes & $133(14.1)$ \\
\hline Cardiac & & Fatigue & \\
\hline No & $886(93.8)$ & No & $714(75.6)$ \\
\hline Yes & $59(6.2)$ & Yes & $231(24.4)$ \\
\hline Hypertension & & SOB & \\
\hline No & $691(73.1)$ & No & $689(72.9)$ \\
\hline Yes & $254(26.9)$ & Yes & $256(27.1)$ \\
\hline Type II Diabetes Mellitus & & Headache & \\
\hline No & $770(81.5)$ & No & $793(83.9)$ \\
\hline Yes & $175(18.5)$ & Yes & $152(16.1)$ \\
\hline Asthma & & $\begin{array}{l}\text { ACEIs, ARBs and/or NSAIDs } \\
\text { use }\end{array}$ & \\
\hline No & $893(94.5)$ & No & $830(87.8)$ \\
\hline Yes & $52(5.5)$ & Yes & $115(12.2)$ \\
\hline Fever & & COVID-19 Severity & \\
\hline No & $739(78.2)$ & Mild & $374(39.6)$ \\
\hline Yes & $206(21.8)$ & Moderate & $299(31.6)$ \\
\hline Cough & & Severe & $272(28.8)$ \\
\hline No & $445(47.1)$ & Outcome & \\
\hline Yes & $500(52.9)$ & Alive & $900(95.2)$ \\
\hline Sore throat & & Dead & $45(4.8)$ \\
\hline No & $827(87.5)$ & & \\
\hline Yes & $118(12.5)$ & & \\
\hline
\end{tabular}

Table 1: Socio-demographic and clinical characteristics $(n=945)$ 
Table 2: Comparison of socio-demographic and clinical characteristics based on drug use history $(\mathrm{n}=945)$ 


\begin{tabular}{|c|c|c|c|}
\hline \multirow[t]{2}{*}{ Variable } & \multicolumn{2}{|c|}{ ACEIs, ARBs and/or NSAIDs use } & \multirow[t]{2}{*}{ p-value } \\
\hline & No $(n=830)$ & Yes $(n=115)$ & \\
\hline \multicolumn{4}{|c|}{ Age category (in years) } \\
\hline$<30$ & $246(29.6 \%)$ & $4(3.5 \%)$ & $<0.0001 *$ \\
\hline $30-39$ & $168(20.2 \%)$ & $9(7.8 \%)$ & \\
\hline $40-49$ & $153(18.4 \%)$ & $17(14.8 \%)$ & \\
\hline $50-59$ & $94(11.3 \%)$ & $31(27.0 \%)$ & \\
\hline$\geq 60$ & $169(20.4 \%)$ & $54(47.0 \%)$ & \\
\hline \multicolumn{4}{|l|}{ Sex } \\
\hline Female & $332(40.0 \%)$ & $40(34.8 \%)$ & 0.283 \\
\hline Male & $498(60.0 \%)$ & $75(65.2 \%)$ & \\
\hline \multicolumn{4}{|l|}{ Fever } \\
\hline No & $642(77.3 \%)$ & $97(84.3 \%)$ & 0.088 \\
\hline Yes & $188(22.7 \%)$ & $18(15.7 \%)$ & \\
\hline \multicolumn{4}{|l|}{ Cough } \\
\hline No & $385(46.4 \%)$ & $60(52.2 \%)$ & 0.244 \\
\hline Yes & $445(53.6 \%)$ & $55(47.8 \%)$ & \\
\hline \multicolumn{4}{|l|}{ Sore throat } \\
\hline No & $726(87.5 \%)$ & $101(87.8 \%)$ & 0.914 \\
\hline Yes & $104(12.5 \%)$ & $14(12.2 \%)$ & \\
\hline \multicolumn{4}{|l|}{ Runny nose } \\
\hline No & $767(92.4 \%)$ & $108(93.9 \%)$ & 0.564 \\
\hline Yes & $63(7.6 \%)$ & $7(6.1 \%)$ & \\
\hline \multicolumn{4}{|l|}{ Chest pain } \\
\hline No & $706(85.1 \%)$ & $96(83.5 \%)$ & 0.657 \\
\hline Yes & $124(14.9 \%)$ & $19(16.5 \%)$ & \\
\hline \multicolumn{4}{|l|}{ Myalgia } \\
\hline No & $712(85.5 \%)$ & $100(87.0 \%)$ & 0.735 \\
\hline Yes & $118(14.2 \%)$ & $15(13.0 \%)$ & \\
\hline \multicolumn{4}{|l|}{ Arthralgia } \\
\hline No & $712(85.8 \%)$ & $100(87.0 \%)$ & 0.735 \\
\hline Yes & $118(14.2 \%)$ & $15(13.0 \%)$ & \\
\hline \multicolumn{4}{|l|}{ Fatigue } \\
\hline No & $626(75.4 \%)$ & $88(76.5 \%)$ & 0.797 \\
\hline Yes & $204(24.6 \%)$ & $27(23.5 \%)$ & \\
\hline \multicolumn{4}{|l|}{ SOB } \\
\hline No & $622(74.9 \%)$ & $67(58.3 \%)$ & $<0.0001 *$ \\
\hline Yes & $208(25.1 \%)$ & $48(41.7 \%)$ & \\
\hline
\end{tabular}




\begin{tabular}{|c|c|c|c|} 
Headache & & \\
\hline No & $695(83.7 \%)$ & $98(85.2 \%)$ & 0.685 \\
\hline Yes & $135(16.3 \%)$ & $17(14.8 \%)$ & \\
\hline COVID-19 Severity & & & \\
\hline Mild & $344(41.4 \%)$ & $30(26.1 \%)$ & $<0.0001 *$ \\
\hline Moderate & $268(32.3 \%)$ & $31(27.0 \%)$ & \\
\hline Severe & $218(26.3 \%)$ & $54(47.0 \%)$ & \\
\hline Outcome & & & \\
\hline Alive & $796(95.9 \%)$ & $104(90.4 \%)$ & $\mathbf{0 . 0 1 0 *}$ \\
\hline Dead & $34(4.1 \%)$ & $11(9.6 \%)$ & \\
\hline Length of admission (in days) & $14.5(5.03)$ & $14.4(5.99)$ & $\mathbf{0 . 0 0 2 *}$ \\
\hline
\end{tabular}

Table 2: Comparison of socio-demographic and clinical characteristics based on drug use history $(n=945)$ 